Document downloaded from:

http://hdl.handle.net/10251/151295

This paper must be cited as:

Moreno-Navarro, P.; Pérez-Aparicio, JL.; Gómez-Hernández, JJ. (2017). Optimization of pulsed thermoelectric materials using simulated annealing and non-linear finite elements. Applied Thermal Engineering. 120:603-613. https://doi.org/10.1016/j.applthermaleng.2017.04.036

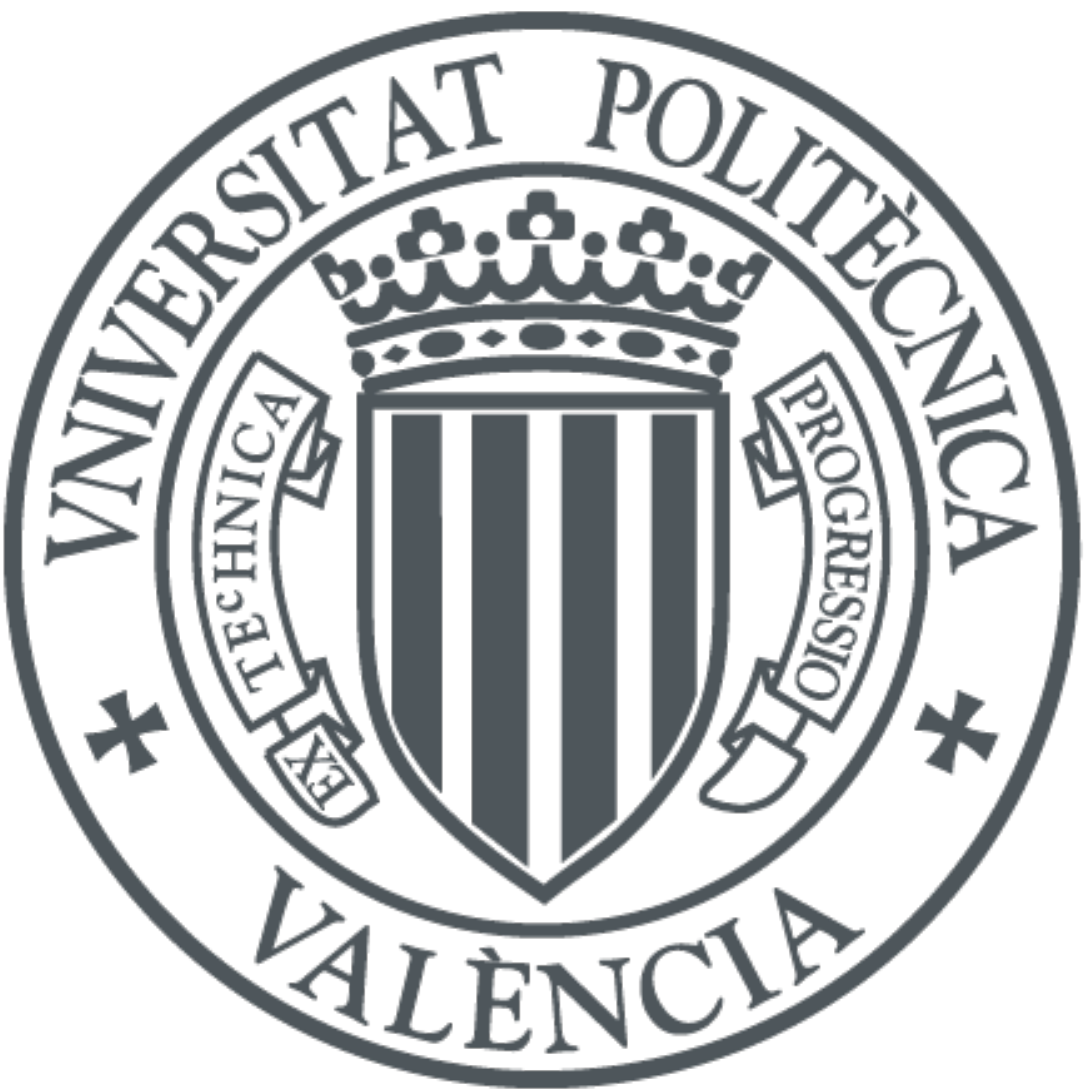

The final publication is available at

https://doi.org/10.1016/j.applthermaleng.2017.04.036

Copyright Elsevier

Additional Information 


\title{
Optimization of pulsed thermoelectric materials using simulated annealing and non-linear finite elements.
}

\author{
P. Moreno-Navarro ${ }^{\mathrm{a}}$, J.L. Pérez-Aparicio ${ }^{\mathrm{b}}$, J.J. Gómez-Hernández ${ }^{\mathrm{c}}$ \\ ${ }^{a}$ Sorbonne Universités - Université de Technologie Compiègne, Laboratoire Roberval de Mécanique, Chaire de Mécanique*, France \\ ${ }^{b}$ Department of Continuum Mechanics $\mathcal{E}$ Theory of Structures, Universitat Politècnica de València, Spain \\ ${ }^{c}$ Research Institute of Water and Environmental Engineering, Universitat Politècnica de València, Spain
}

\begin{abstract}
The objective of this work is to determine the optimal shape, gains and duration of an electric pulse applied to a Peltier cell, together with the length of the thermoelectric to maximize cooling while minimizing electric consumption. For this purpose, a fully coupled, multiphysics, dynamic finite-element model, which solves for the thermal, electric and mechanical fields is used. Because of the demanding computing requirements of the optimization process, a special mesh is designed and a convergence analysis is carried out before using the multiphysics model. The highly nonlinear optimization is done by simulated annealing, a heuristic algorithm in the Markov chain Monte-Carlo family. A preliminary parametric investigation is presented, analyzing the impact of some of the parameters. The results of this preliminary analysis help to understand the effect of the different shapes in the evolution of the cold face temperature. Some of these results are expected and have already been discussed elsewhere, but others can only be explained after further analysis and a full system modeling. Pulse optimization is multiobjective and multiparametric, i.e., it can consider several targets such as maximizing the cooling temperature, the cooling duration or others. The trade-offs between the different targets are studied. In all cases, stresses inside the thermoelement are examined at all points, and the pulses must meet the restriction that an equivalent stress is not above the allowable value.
\end{abstract}

Keywords: Pulsed thermoelectrics, non-linear dynamic finite elements, multiphysics, pulse shape optimization, simulated annealing, multiobjective-multiparameter

\section{Nomenclature}

$\begin{aligned} n & \text { Nodes per edge } \\ l & \text { Length } \\ x & \text { Spatial coordinates } \\ P & \text { Pulse gain } \\ T & \text { Temperature } \\ t & \text { Time } \\ \theta & \text { Annealing temperature } \\ \lambda_{a} & \text { Reduction factor of } \theta \\ K & \text { Maximum number of iterations } \\ S_{p} & \text { Stopping number } \\ O & \text { Objective function } \\ \boldsymbol{p} & \text { Set of input parameters } \\ \boldsymbol{\varphi} & \text { Set of weight parameters } \\ \mathcal{T} & \text { Equivalent stress } \\ \sigma & \text { Standard deviation } \\ \mathcal{S} & \text { Optimization goal }\end{aligned}$

Sub-, Supra-indices

$i \quad$ Spatial direction, counter
Prescribed property for $\square$

$h \quad$ Hot side $(T)$, holding $(t)$

$c$ Cold side

0 Reference

ac Accepted

rj Rejected

$p$ Pulse

pp Post-pulse

op Optimal

css Cold face at steady-state

mn Minimum

$m x \quad$ Maximum

$I, I I, I I I$ Principal directions

tr Tresca

ad Admissible

\section{Introduction}

In spite of the many papers published on pulsed thermoelectrics (PT), the study of the pulse shape itself has 
attracted limited attention and the most common shape is a step (in reality, slightly trapezoidal), see for instance [1]. Given the advances in quality and price of electric and electronic control equipment, it is pertinent to improve PT's performance and consumption.

The purpose of the present paper is to determine pulse optimal shapes for thermoelectric material (TM) refrigeration with Peltier cells; these pulses will not have a predefined shape but will be within a bounding envelop. The shape is closely related with the thermoelement (TE) length $l$ (of "boxed" parallelepipedal geometry), and the duration of the pulse itself; both of them are input parameters to the optimization algorithm. It will be shown that suitable (although not easy to guess) combinations of shapes and lengths can substantially improve the performance of the Peltier cell, for eight different scenarios.

Recently, [2] considered several predetermined pulses (step, linear and quadratic) to differentiate the overcooling of micro and nanoscales, deciding by inspection, the two best pulses for each scale. The Maxwell-Cattaneo constitutive equations and dissipative flows were considered, with thermal and electric conductivities as a function of the layer thickness. Also, [3] carried out a parametric study of pulses for refrigeration; no elastic coupling was considered since the models were purely thermoelectric, although the boundary conditions $(\mathrm{BC})$ were realistic with convection in the hot face and constant prescribed heat flux in the cold face. The model was validated and the differences between constant and variable properties studied. No optimization algorithm was applied, instead conclusions were drawn from the extensive parametric set of cases. Another parametric study was presented in [4] analyzing, in a rather simple manner for constant pulses, the influence of parameters such as pulse gain and duration, using an one-dimensional (1D) model. Constant pulses were also analyzed in [5] but an experimental parametric investigation instead of a numerical model was presented. This work was focused more on boundary conditions than on pulse parameters and the pulse train influence was shown as well.

Other types of optimizations have been published. For instance, [6] optimized the material properties and the ratio of TE area and length using simple analytical models and experimental results. In [7] and [8], cooling applications were optimized, in the former with electric network models and finite elements (FE) for refrigerators, and in the latter with $1 \mathrm{D}$ analytical formulae for radiating air conditioning.

In [9], ANSYS was used on a novel and predetermined pulse shape for a practical application; the opti- mization goals were similar to the ones of the present article. The work presented a parametric study on pulse gain, duration for basic pulse shapes (constant, triangular, ramps), quantifying the overcooling produced by the pulse with respect to the stationary state.

An optimization of a two-stage TM refrigerator with a 1D numerical method was presented in [10], but the optimization was not for the pulse itself but for the positions of the thermocouple (TC). Analytical formulae were developed to calculate the optimal intensity, and based on these, the optimal coefficient of performance (COP) and extracted heat were determined; this was achieved varying the number of TCs and the hot face temperature $T_{h}$ although in static operation. The articles [11] and [12] were also related with two-stage refrigerators. In the former, a multiparametric studychanging the heat source, sink and current-is developed, comparing it with experimental results. In the latter, a more realistic model with convection is run for another multiparametric study, in which the pulse duration, gain and shape as well as basic parameters of the geometry are varied.

Finally, [13] developed a simplified 1D model (not including the Thomson effect) based on a Fourier sums of eigenvalues, to analytically predict the cold face temperature $T_{c}$. The next step was to optimize the distributions of the Seebeck coefficient $\alpha$ and electric conductivity $\gamma$ along the TE (as in graded materials) under a constant pulse. Empirical expressions for maximum overcooling and time to reach it as a function of the pulse gain were developed.

In the current work, it will be assumed that, both for steady and transient states, the intensity is the same for all TCs of the cell; therefore, a single TC is discretized under appropriate BC. The cold face is subjected to a cooling optimal intensity calculated from semi-analytical formulae taken from [14] prior to the application of the pulse. The hot face is considered attached to an infinite medium so that its temperature remains constant at $\bar{T}_{h}=50{ }^{\circ} \mathrm{C}$. Regarding the mechanical $\mathrm{BC}$, both hot and cold faces are mechanically hinged. To reduce the FE mesh, two planes of symmetry and a plane of repetition will be applied.

The FE model, developed in [15], [16] and [17], has been shown to be very efficient for the modeling of cooling using Peltier cells. FEAP, a research code with special elements developed by [18] is used.

The optimization model is heuristic in nature, which is both an advantage and a shortcoming; an advantage, because it will search for global optimal solutions, avoiding local optima, and a shortcoming, because it requires many runs of the FE model needing much $\mathrm{CPU}$ 
time to reach the optimum. This optimization algorithm was chosen for its power to minimize complex functions involving non-linearities, fully coupled processes, system dynamics and 3D geometry without imposing any restrictions in the potential outcomes.

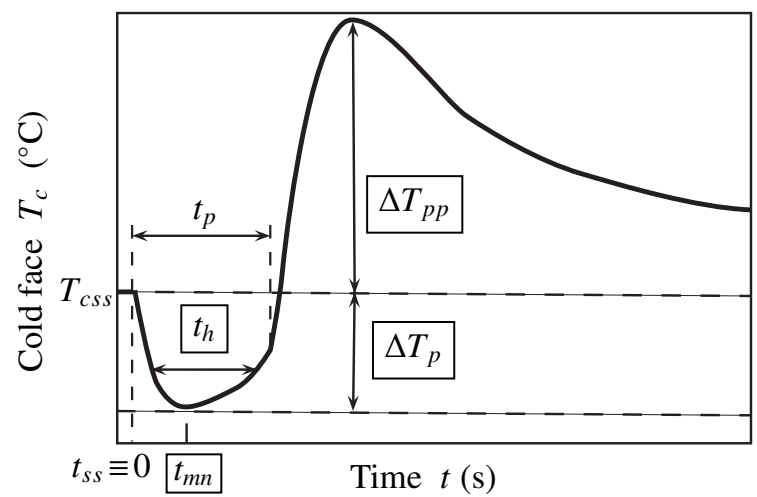

Figure 1: Schematic diagram of temperature evolution at the thermoelement cold face subjected to a pulse of undetermined shape. Boxed symbols indicate optimization targets.

In Fig. 1, a conceptualization of the temperature $T_{c}$ evolution at the cold face is shown. From the steadystate $T_{c s s}$ and after a pulse of gain $P$ is applied at time $t_{s s}$ (set to zero), the temperature descends drastically until it reaches a minimum $T_{c s s}-\Delta T_{p}$ at time $t_{m n}$; this is the transient overcooling phase. Close to the end of the pulse, of duration $t_{p}$, the temperature bounces back and reaches a value $T_{c s s}+\Delta T_{p p}$ (both $\Delta T_{p}$ and $\Delta T_{p p}$ are positive); this is the transient overheating phase. If no other pulse is introduced, the temperature slowly lowers back to the initial $T_{c s s}$. The description and related dynamic calculations of a PT have thoroughly been studied in [14].

Before launching the optimization algorithm and its thousands of runs, a convergence study of the fully coupled FE code is performed in Section 2. The usefulness of this convergence study is to find the mesh with the minimum number of elements that gives reasonable results, for the mechanical, thermal and electric fields.

A parametric study is carried out in Section 3 with simple pulse geometries, as defined by one or two straight or curved lines; also, the different goals are described in this section. This parametric study will help us understand why some pulse shapes are beneficial or harmful for a given goal, and will provide critical knowledge for the interpretation of the final shapes.

The optimization algorithm is described in Section 4. Its application will permit one to develop optimal pulses automatically (not based on physical or engineering insight), which can provide unsuspected and virtually impossible-to-guess solutions. Finally, in Section 5 optimal pulses are computed for eight cases, the first four aiming at the optimization of a single goal, and the other four at the joint optimization of several goals. The resulting pulses are then described and analyzed.

\section{Finite Element model}

The governing equations, and the special finite element developed, are thoroughly described in [15] (general multicoupled formulation) and [14] (pulse dynamics) and will not be repeated here. However, given the high computational requirements of the optimization algorithm, a convergence study for various FE meshes is presented to determine the minimum number of elements that achieves a good compromise between error and computation time.

The structured mesh is parametrized with a variable number of elements in some directions and materials, except for $\mathrm{Cu}, \mathrm{Al}_{2} \mathrm{O}_{3}$ and solder $\mathrm{Sn}_{2} \mathrm{~Pb}_{3}$ in $x_{3}$, which will be discretized considering only one element in the vertical direction (see Fig. 2). For the solder, and to increase the realism of the simulation, a small element is added outside of the TE line along $x_{1}$ to simulate the burrs that often protrude after the welding process. Given the low elastic modulus of tin-lead and in spite of its very low thickness (viz. [19]), it is important to include these burrs in the simulation, since they can relieve or concentrate the stresses close to the TE corners.

Only the stresses in the TE will be monitored, since it is the only active material. The stresses of the other materials can be high due to their high relative stiffness, entering in plastic regime and affecting the TE in their junctions. This plasticity is ignored in the present work, but in an article recently published [20] it is considered in all materials of the cell.

The main parameter that defines the level of refinement is $n_{3}$, the TE number of nodes in the $x_{3}$ direction. The choice of this number also depends on $l$, a critical dimension for PTs. To capture stress concentrations at corners (viz. [14]), a maximum height along $x_{3}$ of $3 \times 10^{-3} \mathrm{~m}$ for each quadratic 27 -node finite element will be set for the variable $l$ (half of this height for linear 8-node elements).

The simulation of the electric and thermal fields requires a discretization of very few elements and nodes due to their vectorial nature, but the mechanical field is much more demanding due to its tensorial nature and the appearance of stress concentrations. Figure 3 shows the maximum Tresca stress $\mathcal{T}_{t r}=$ $\max \left(\left|\mathcal{T}_{I}-\mathcal{T}_{I I}\right|,\left|\mathcal{T}_{I I}-\mathcal{T}_{I I I}\right|,\left|\mathcal{T}_{I}-\mathcal{T}_{I I I}\right|\right)$ inside the TE, 


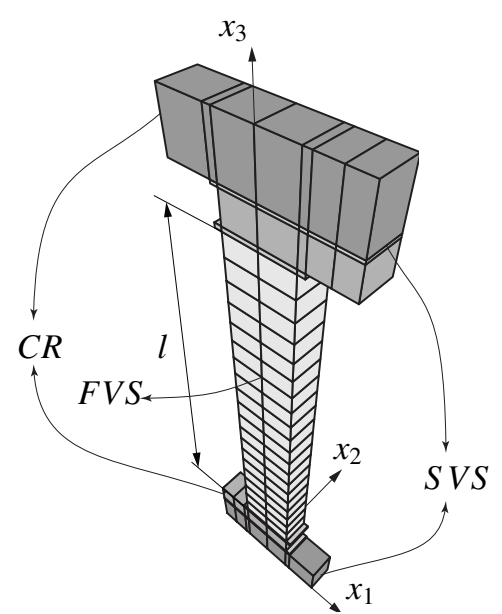

Figure 2: Finite element mesh for $1 / 4$ thermocouple: $F V S$, plane of $x_{1}-x_{3}$ vertical symmetry with itself; $C R$ two "cuts" for repetitions with other thermocouple; $S V S$, plane of $x_{2}-x_{3}$ vertical symmetry with the other thermoelement. Length $l=6 \times 10^{-3} \mathrm{~m}$ with $n_{3}=20$ elements; from higher to lower grey: $\mathrm{Al}_{2} \mathrm{O}_{3}, \mathrm{SnPb}, \mathrm{Cu}, \mathrm{Bi}_{2} \mathrm{Te}_{3}$ materials.

in which roman number subindices mean principal directions. This is an equivalent stress that combines several of the stress tensor components into a unique value, and it is based in the classical failure criterion of Tresca, very similar to that of Von Mises.

These stresses are calculated at any integration point of the TE at steady-state for different refinements and with $l=6 \times 10^{-3} \mathrm{~m}$. It can be observed that the maximum stresses converge quickly to a stable value as a function of the number of elements along the horizontal axes. For both 8-node and 27-node elements, a discretization of 9 nodes per edge is enough to approximate the maximum stress, even 5 nodes per edge could be considered acceptable given the small difference of $2 \mathrm{MPa}$ between 5 and 9 nodes. The oscillation in the maximum stress observed for the lower values of $n_{3}$ when using the 27-node elements is probably due to the imprecision resulting from the proximity of a rather thin element in the TE to the thick element in $C u$. The 8-node element tends to predict slightly higher stresses than the 27-node one due to the simplicity of the linear FE formulation.

The vertical discretization is critical and a fine mesh is needed in this direction to properly approximate maximum stresses. Fig. 4 shows the convergence of the maximum Tresca stress as a function of $n_{3}$ at the three significant moments defined before in Fig. 1. For $n_{3}>$ 41 , the stress increase is very small and convergence has been achieved. In Fig. 4, the calculated stresses for the 27-node are in general higher than those of the 8-node,

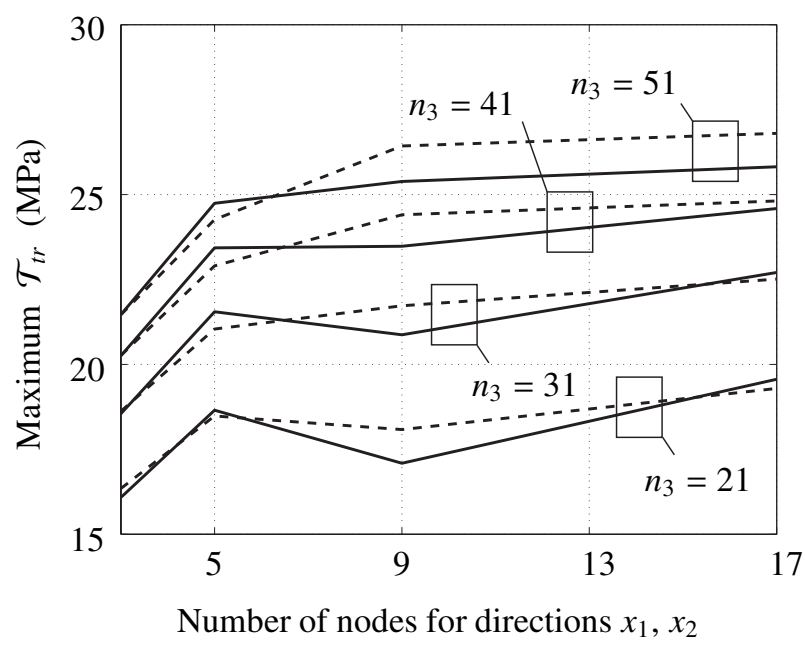

Figure 3: Maximum Tresca stress (on any point at steady-state) as a function of lateral thermoelement refinement along $x_{1}$ or $x_{2}$, for different $n_{3}$ along $x_{3}$, see Fig. 2. Solid line for 27-node 3D isoparametric finite element, dashed line for 8-node elements.

since the integration Gauss point of the former is much closer to the corner than that of the latter (viz. [21]). The difference is more noticeable when dynamics are present, in particular at pulse removal, but for high $n_{3}$ the results almost coincide for both elements. It was also found that refinements in other materials such as $\mathrm{Cu}$ or $\mathrm{Al}_{2} \mathrm{O}_{3}$ have little influence in the approximation of the maximum Tresca stress inside the TE.

Finally, 5 nodes per edge in the horizontal directions, and 41 nodes in the vertical direction are chosen; in reality only 3 nodes will be used in the $x_{2}$ direction due to symmetry considerations. Also, for only a slightly higher computational cost (with the same number of degrees of freedom) the more accurate 27-node element will be chosen (viz. [21]). The concluding mesh, comprising 88 elements, 1686 nodes and 6755 degrees of freedom (five per node: three displacements plus temperature and voltage) is shown in Fig. 2. The computation time of a single run ranges from $170 \mathrm{~s}$ for $n_{3}=11$ to $320 \mathrm{~s}$ for $n_{3}=61 \mathrm{in}$ an Intel Core $17-4930 \mathrm{~K} \mathrm{CPU}$ running at $3.40 \mathrm{GHz}$ and compiled in gfortran for linux, version 4.6.3.

\section{Parametric study}

A review of practical applications taken from the literature: [22], [23], [24], indicates that an optimal pulse should, at least, aim to the following four goals $\mathcal{S}_{j}$ :

- $\mathcal{S}_{1}$ : minimization of $1 / \Delta T_{p}$, to maximize the overcooling between $T_{c s s}$ and the minimum $T_{c}$. 


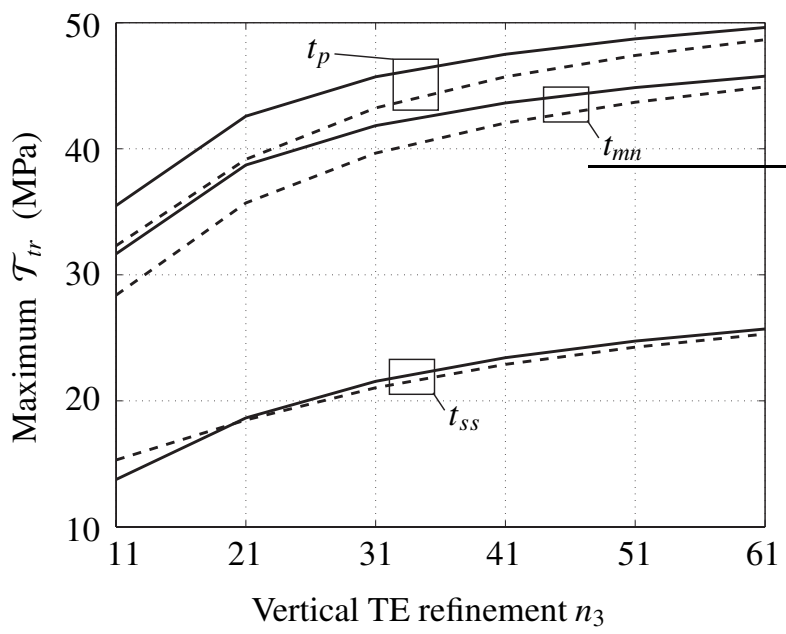

Figure 4: Convergence of maximum Tresca stress at any point for thermoelement refinement $n_{3}$ along $x_{3}$, see Fig. 2. Curves for steadystate $t_{s s} \equiv 0$, maximum overcooling $t_{m n}$ and pulse removal $t_{p}$. Solid line for 27-node, dashed for 8-node 3D isoparametric finite elements.

- $\mathcal{S}_{2}$ : minimization of $t_{m n}$, the time to reach the min$\operatorname{imum} T_{c}$.

- $\mathcal{S}_{3}$ : minimization of $\Delta T_{p p}$, the overheating between $T_{c s s}$ and maximum $T_{c}$.

- $\mathcal{S}_{4}$ : minimization of $1 / t_{h}$, to maximize the holding time during which the overcooling is at least $0.8 \Delta T_{p}$.

Each goal is directly or inversely related to one of the four targets: $\Delta T_{p}, t_{m n}, \Delta T_{p p}, t_{h}$; the last one is often called overcooling uptime. The overheating $\Delta T_{p p}$ is a detrimental effect for PTs not only because increases the temperature of the cold face but also because large values induce a long time of recovery to the second steadystate $T_{\text {css }}$.

A parametric study is performed in this section to have an a-priori estimate of the results that will be obtained by the optimization process, in particular what type of pulse is best for each goal or combinations of them. The predefined pulse shapes shown in Fig. 5 are considered; some of them have been already studied in the past and some are new. Other important parameters and their range of values to be analyzed in the parametric study are: the TE length, $l \in[2,7] \mathrm{mm}$ at increments of $1 \mathrm{~mm}$, the pulse gain $P \in[1.5,3.5]$ at increments of 0.5 and the pulse duration $t_{p} \in[2,8] \mathrm{s}$ at increments of $2 \mathrm{~s}$. The 720 combinations of parameters $(6$ shapes times 6 TE lengths times 5 pulse gains times 4 pulse durations) are run and the values of the four goals $\mathcal{S}_{j}$ computed.

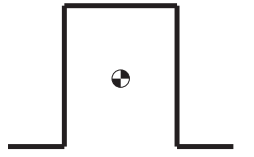

$\mathcal{A}$

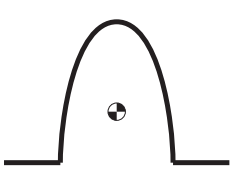

$\mathcal{D}$

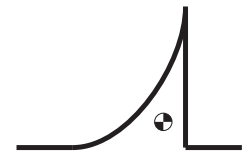

$\mathcal{B}$

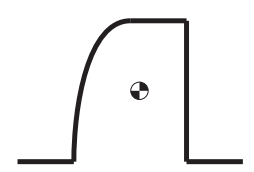

$\mathcal{E}$

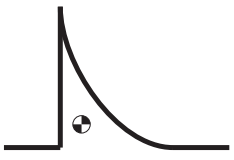

C

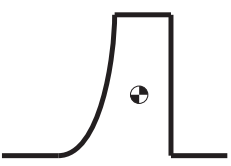

$\mathcal{F}$
Figure 5: The six generic pulse shapes used in the parametric study: $\mathcal{A}$ : Rectangular, $\mathcal{B}$ : Quadratic, $\mathcal{C}$ : Inverse Quadratic, $\mathcal{D}$ : Sinusoidal, $\mathcal{E}$ : Sinusoidal-Rectangular, $\mathcal{F}$ : Quadratic-Rectangular. Pulses' centers of gravity indicated by crossed circles.

In Fig. 6, the transient evolutions of $T_{c}$ for the pulses of Fig. 5 are shown for a representative length, gain and pulse duration. As published elsewhere, very different pulses induce radically different responses, during the pulse itself and during the post pulse. In spite of the large duration considered, some of the evolutions are interrupted at pulse removal (remarkably $\mathcal{B}$ ), meaning that a longer duration would only slightly increase $\Delta T_{p}$, but surely also increase $\Delta T_{p p}$. The contrary can be said about pulses that reach their minimum $T_{c}$ substantially before the end of the pulse ( $\mathcal{A}$ and $C$ ): a shorter duration would improve their performance.

A smooth introduction of the pulse is convenient for overcooling, since the bulk of the Joule effect will be generated later and then take longer to reach the cold face by conduction. In fact, the best pulse to maximize $\Delta T_{p}$ is $\mathcal{F}$ closely followed by $\mathcal{D}$ and $\mathcal{E}$ (pulse $\mathcal{B}$ will be commented later). The evolutions of $\mathcal{D}$ and $\mathcal{E}$ coincide during the first instants, since the pulses are equal in their first half.

On the contrary, $\mathcal{A}$ and $C$ have an initial sharp pulse and thus they produce the worst overcooling of all pulses. Of the two of them, $C$ yields the lower value as a result of its smaller Peltier effect, proportional to the electric energy of the pulse as measured by the area under it. The Joule effect is also proportional to the pulse area, but quadratically: there is then a competition between both effects that depends on several parameters. In the figure it can be seen that the overcooling of $C$ stops at $2 \mathrm{~s}$ for lack of extra energy while that of $\mathcal{A}$ continues at least $1,5 \mathrm{~s}$ more.

Again, the more sudden the introduction of the pulse at the first instant, the higher the slope of the $T_{c}$ evolution. The minimum temperature is also reached the fastest for pulses that start with a sharp impulse, for this 
reason $C$ is only advantageous for $t_{m n}$.

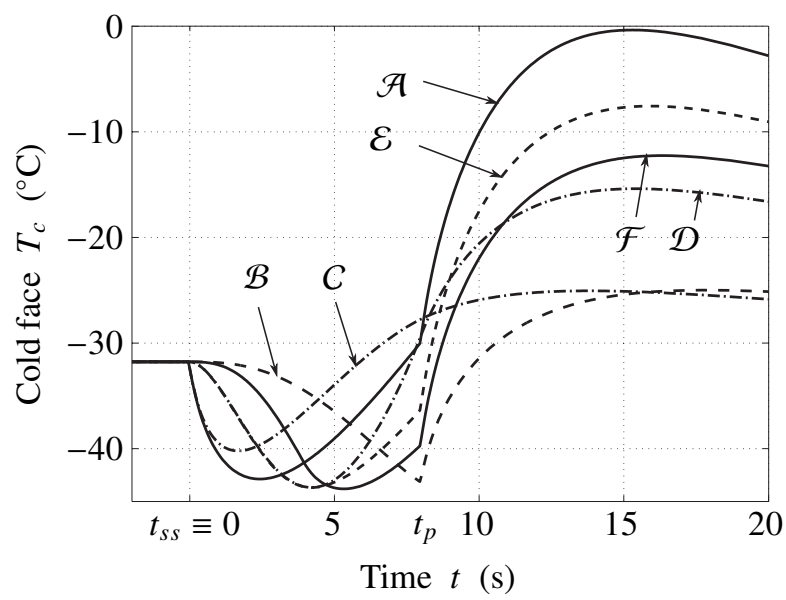

Figure 6: Transient evolution of the cold face temperature for the generic pulses of Fig. 5 for thermoelement length $5 \times 10^{-3} \mathrm{~m}$, maximum pulse gain $P=2.5$ and pulse duration $t_{p}=8 \mathrm{~s}$.

The best pulses to minimize the overheating $\Delta T_{p p}$ are $\mathcal{B}$ and $\mathcal{C}$, while the worst ones are $\mathcal{A}$ and $\mathcal{E}$. This behavior is easily explained considering again that the Joule effect and, consequently the overheating, is proportional to the pulse area square: comparing the similar shapes $\mathcal{D}$ and $\mathcal{E}$, the first produces a smaller overheating due to its smaller area than the second one.

Finally, the pulses that produce the best uptime are $\mathcal{A}$, $\mathcal{E}$ and $\mathcal{F}$ for the reasons that will be better understood in Section 5 based on the results of the optimization exercise. It can be observed that a long $t_{p}$ will improve $t_{h}$ but it will go against a good $\Delta T_{p}$, and viceversa.

The position of the pulse center of gravity $(\mathrm{cg}$, see Fig. 5) is related to the area under the pulse, and is the other important factor explaining the $T_{c}$ evolution. In general, the closer the cg to the pulse top, the higher $\Delta T_{p}$ and $\Delta T_{p p}$, see shapes $\mathcal{A}, \mathcal{E}, \mathcal{F}$ and $\mathcal{D}$. As explained, minimum overheating occurs for $C$, since it is the pulse with the cg closest to $P=1$. The time $t_{m n}$ is related to the position of the $\mathrm{cg}$ along the time axis: when the $\mathrm{cg}$ is close to the pulse beginning, as is the case for $C$, all the energy is quickly injected into the system and the minimum $T_{c}$ is reached fast although with a low value; whereas when the $\mathrm{cg}$ is close to the end of the pulse, as is the case for $\mathcal{B}$, the minimum $T_{c}$ is reached more slowly. Note that if the $C$ curvature would be convex (decreasing slowly after the spike) instead of concave, the cg would move towards the right and top, its $\Delta T_{p}$ should increase and $\Delta T_{p p}$ decrease.

If any of the previous trends is considered alone, wrong conclusions can be drawn. For instance, it can be thought that $\mathcal{B}$ should have a low $\Delta T_{p}$ since it introduces low energy (cg towards the bottom), but the result is among the best since this introduction is smooth and consequently the cg falls off towards the right.

After analyzing the values of the parameters associated with the four targets, the instances that produce the best values for each goal are identified and listed in Table 1. There was no optimization involved, simply a combinatorial search. The maximum overcooling is a significant $50 \%$ higher than that of the steady-state; the minimum temperature is reached as early as $3.3 \%$ of $t_{p}$, the optimal overheating is close to zero and the best holding time is as long as $77 \%$ of $t_{p}$. As expected, the maximum $\Delta T_{p}$ and $t_{h}$ happen for the longest possible $t_{p}$; also the maximum $\Delta T_{p}$ and the minimum $t_{m n}$ are obtained for the pulses with the highest $P$.

Analyzing the TE lengths, it can be noted that the larger $l$, the longer for the Joule effect to overcome the Peltier effect near the cold face, therefore, the maximum $l$ is the one that maximizes the $\Delta T_{p}$. The optimal $l$ is close to its minimum value for $C$ when targeting the minimization of $t_{m n}$ because the slope of $T_{c}$ at the beginning is the highest and therefore the Joule effect arrives to the cold face sooner. The relationship between a high $T_{c}$ slope and a short $l$ is due to the high optimal intensity at steady-state $I_{o p}$, which is proportionally inverse to $l$, see [14] and Table 1.

\begin{tabular}{|c|c|c|c|c|c|c|c|}
\hline Target & Shp. & $t_{p}$ & $P$ & $l$ & $I_{o p}$ & Val. & $\mathcal{T}_{t r}$ \\
\hline \hline$\Delta T_{p}$ & $\mathcal{F}$ & 8 & 3.5 & 7 & 0.76 & 16.1 & 72.8 \\
\hline$t_{m n}$ & $\mathcal{C}$ & 2 & 3.5 & 2 & 2.62 & 0.27 & 77.6 \\
\hline$\Delta T_{p p}$ & $\mathcal{C}-\mathcal{B}$ & 2 & 1.5 & 6 & 0.89 & 0.27 & 24.4 \\
\hline$t_{h}$ & $\mathcal{A}$ & 8 & 1.5 & 5 & 1.06 & 6.16 & 31.6 \\
\hline
\end{tabular}

Table 1: Optimal pulse shapes and corresponding target values found by combinatorial search. Temperature in ${ }^{\circ} \mathrm{C}$, time in $\mathrm{s}$, length in $\mathrm{mm}$, electric intensity in A and Tresca equivalent stress in MPa.

None of these pulses is able to produce good values for the four targets simultaneously; in fact, some of these targets are contradictory among themselves. For instance, the high energy of $\mathcal{A}$ will produce high overcooling but also high overheating.

Some of the pulses give very good results but they induce stresses above the maximum allowable $\mathcal{T}_{a d}=$ $60 \mathrm{MPa}$ taken from [25]; the values are reported in the last column of Table 1. Stresses will be maximum in general at pulse removal when they almost double those of the steady-state (see Fig. 4). This is logical since an increase of electric intensity implies a strong increase of temperature and consequently of stresses (the reference temperature of $20^{\circ} \mathrm{C}$ remains constant). 
In Section 5 the optimization will automatically include restrictions for the maximum allowable stress. But no restrictions will be introduced with respect to the maximum temperature allowable, since the values observed in this parametric study appear reasonable for all pulse shapes and the present PT configuration.

\section{Simulated Annealing}

Simulated Annealing (SA), viz. [26], is a heuristic global optimization method based on Markov chains that minimizes a given multiobjective function $O$. The name is inspired by the thermal heat treatment called annealing: during the cooling phase of a steel forge, the steel is reheated at some moments of the cooling process to ensure the relieve of residual stresses; the metallic grains will attain, in this way, a relaxed configuration of the lowest energy (global minimum).

The main idea of this numerical method is to continually perturb the input parameters, until $O$ reaches a global minimum. Every perturbation that reduces $O$ is accepted, but to avoid that the optimization gets trapped at a local minimum, certain unfavorable perturbations (i.e., which do not reduce $O$ ) are also accepted.

Several variables define the algorithm:

- $\theta$ : “annealing temperature", $\theta_{0}$ initial value.

- $\lambda_{a}$ : reduction factor of $\theta$.

- $K_{\mathrm{rj}}$ : maximum number of rejected iterations, $i_{r}$, before modifying annealing temperature.

- $K_{\mathrm{ac}}$ : maximum number of accepted iterations, $i_{a}$, before modifying annealing temperature.

- $S_{p}$ : stopping number indicating the maximum number of times that $K_{\mathrm{rj}}$ can been reached.

- $O, \tilde{O}$ : objective function value for the previous iteration, and for the current parameter proposal.

The temperature reduction factor is bounded by $0<$ $\lambda_{a}<1$ and the typical $S_{p}$ is 2 or $3 . K_{\mathrm{ac}}$ and $K_{\mathrm{rj}}$ are often taken as 10 and 100 times the number of input parameters [26], respectively; once any of these values is reached, $\theta$ is reduced by $\lambda_{a}$ and the corresponding counter, $i_{a}$ for accepted iterations, or $i_{r}$ for rejected iterations, is reset to zero.

At the initialization step of SA, the $O$ is evaluated for a proposed set of input parameters $\boldsymbol{p}$, the values of which are set by the user or drawn randomly from predefined probability distribution functions; this first value of $O$ is saved for posterior evaluation of the SA performance. Then, a new parameter proposal is built by selecting at random one of the parameters being optimized, and then drawing a value for this parameter from its probability distribution function (see Section 4.1). Then, the value of the objective function is recalculated, $\tilde{O}$.

If $\tilde{O} \leq O$, the proposal is always accepted (it improves the value of the objective function), and a new iteration is started, $\boldsymbol{p} \leftarrow \tilde{\boldsymbol{p}}$ and $O \leftarrow \tilde{O}$. However, if $\tilde{O}>O$ the proposal will be rejected with a certain probability that depends on the magnitude of the difference $O-\tilde{O}$; the larger this magnitude, the larger the probability of rejection. This probability of rejection also depends on the annealing temperature, the cooler the temperature, the larger the probability. Such a probability can be modeled with a Boltzmann's distribution function:

$$
f_{B}=\exp \left(\frac{O-\tilde{O}}{\theta}\right)
$$

with this distribution, as the number of iteration progresses (and the annealing process cools down) the probability of accepting a proposal that increases the objective function approaches zero.

The most critical decision for an efficient convergence of the algorithm is the choice of $\theta_{0}$ and $\lambda_{a}$. If both are very small, then $\theta$ "cools" rapidly and the SA can be trapped in a local minimum; otherwise, if both are high the convergence may be very slow. In the literature, analytical SA guides can be found to guarantee convergence, see for instance [27].

After some trial and error, the chosen values in the present study are $\lambda_{a}=0.85$ and $\theta_{0}=0.215$. SA ends when it has rejected $S_{p} \cdot K_{\mathrm{rj}}$ iterations.

In Fig. 7 a complete flow diagram of the SA algorithm is presented, using the notation described in this section.

\subsection{Input parameters}

A sketch of the typical piecewise pulse to be calculated is shown in Fig. 8. The input parameters that define this pulse and the TE are:

- Vector of equally-spaced pulse gains $P_{i} \in[1,6]$, $1 \leq i \leq 12$.

- Total pulse duration $t_{p} \in[2,11] \mathrm{s}$.

- TE length $l \in[2.5,7] \times 10^{-3} \mathrm{~m}$ defined in Fig. 2 .

Virtually any practical shape can be found by SA. The vector $\boldsymbol{p}$ that groups the input parameters has entries noted $p_{i}$ with $1 \leq i \leq n=14$. In order to avoid non-plausible shapes, interpolation between pulse gains 


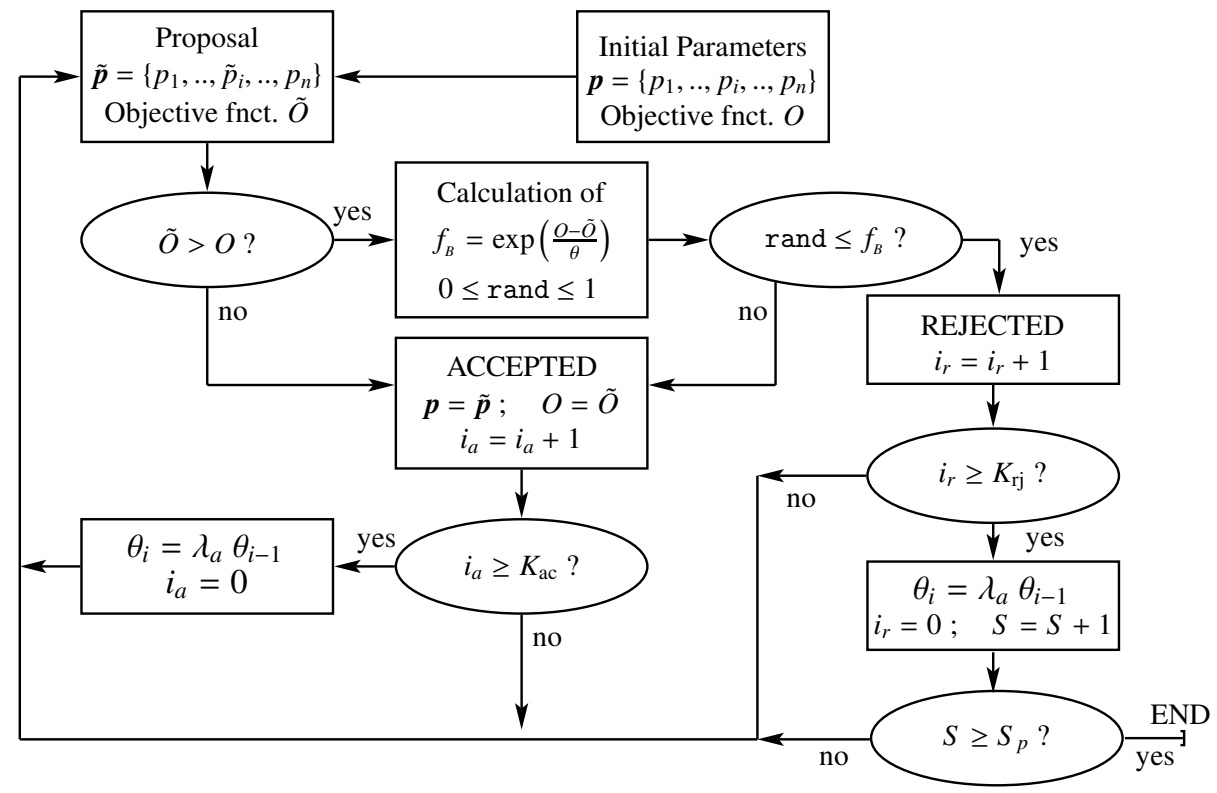

Figure 7: Flow diagram of the Simulated Annealing algorithm applied to optimization of thermoelectric pulses.

is made piecewise with the Matlab ${ }^{\odot}$ function pchip (shape-preserving piecewise cubic) [28]. (Previous tests done using splines resulted in irregular pulse shapes.)

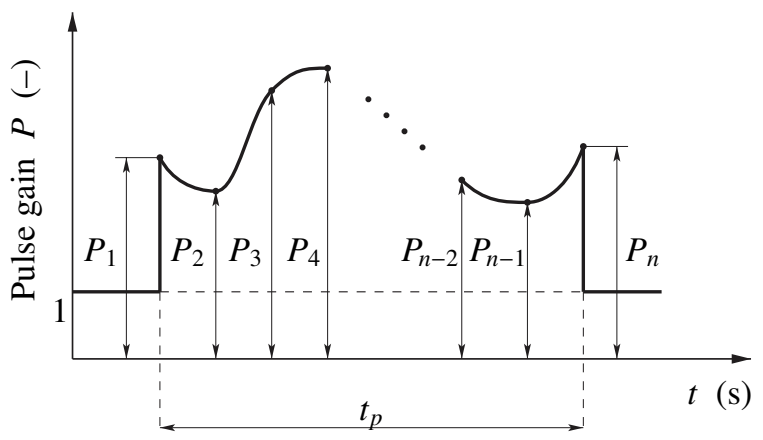

Figure 8: Typical pulse gain evolution vs. time to be calculated by the simulated annealing optimization algorithm.

The objective function $O$ is defined as:

$$
O=\sum_{j=1}^{4} \varphi_{j} \frac{\mathcal{S}_{j}(\boldsymbol{p})-\overline{\mathcal{S}}_{j}}{\sigma_{j}}
$$

where $\varphi=\left\{\varphi_{1}, \varphi_{2}, \varphi_{3}, \varphi_{4}\right\}$ is a set of weights adding up to one defining the relative importance given by the user to each target $\mathcal{S}_{j}$. In addition, $\overline{\mathcal{S}}_{j}$ and $\sigma_{j}$ are the mean and the standard deviation of $n=20$ trial runs with random parameter values in the specified ranges. These statistics are used to normalize the values of the targets ensuring that when considered in the optimization function they have contributions of the same order of magnitude.

The values obtained are listed in Table 2 . The mean of the inverse of maximum overcooling is $1 / 6.5^{\circ} \mathrm{C}^{-1}$, the mean of the time to reach it is $3 \mathrm{~s}$, the mean overheating is $12{ }^{\circ} \mathrm{C}$ and the mean holding time is $0.84 \mathrm{~s}$.

The mean holding time $t_{h}$ is a rather small value and the mean time to reach maximum overcooling $t_{m n}$ is a rather big one. These values are clearly not optimal, the optimal values that will result after SA are quite far from these mean values as will be shown later.

\begin{tabular}{|c|c|c|c|c|}
\hline Goal & 1 & 2 & 3 & 4 \\
\hline \hline$\overline{\mathcal{S}}_{j}$ & $1 / 6.45$ & 3.02 & 11.82 & $1 / 0.84$ \\
\hline$\sigma_{j}$ & 0.066 & 1.82 & 5.012 & 0.6 \\
\hline
\end{tabular}

Table 2: Means and standard deviations to be used to normalize the target values in the optimization equation.

\subsection{Cases}

The weights in Table 3 define the eight cases analyzed. In the first four cases, the optimal pulses for each goal considered alone are determined. In the last four cases, the optimal pulses for different combinations of more than one goal are computed. 


\begin{tabular}{|c|c|c|c|c|}
\hline Case & $\varphi_{1}$ & $\varphi_{2}$ & $\varphi_{3}$ & $\varphi_{4}$ \\
\hline \hline 1 & 1 & 0 & 0 & 0 \\
\hline 2 & 0 & 1 & 0 & 0 \\
\hline 3 & 0 & 0 & 1 & 0 \\
\hline 4 & 0 & 0 & 0 & 1 \\
\hline 5 & $1 / 4$ & $1 / 4$ & $1 / 4$ & $1 / 4$ \\
\hline 6 & $1 / 3$ & $1 / 3$ & $1 / 3$ & 0 \\
\hline 7 & $1 / 3$ & 0 & $1 / 3$ & $1 / 3$ \\
\hline 8 & $1 / 2$ & 0 & $1 / 2$ & 0 \\
\hline
\end{tabular}

Table 3: Optimization function weights for the eight cases analyzed.

In all cases, the initial proposal of parameters are: a pulse of constant $P=2, t_{p}=5.5 \mathrm{~s}$ and $l=4.75 \times 10^{-3}$ $\mathrm{m}$. With this proposal set the FE code is run, the values of the four targets, $\mathcal{S}_{j}$, computed and the first value of $O$ obtained. Then, iterations start selecting randomly one parameter out of the 14 parameters, and proposing a new value for it. This new value is randomly drawn from a cumulative probability distribution function with the following expression:

$$
\operatorname{Prob}\left(p_{i} \leq \tilde{p}_{i}\right)=f\left(\tilde{p}_{i}\right)=\left(\frac{\tilde{p}_{i}-p_{\text {imn }}}{p_{\text {imx }}-p_{\text {imn }}}\right)^{1 / m}
$$

with $p_{\text {imn }}$ and $p_{\text {imx }}$ being the minimum and maximum values of the parameter, respectively, and $m$ a coefficient controlling the skewness of the distribution. For $m=$ 1 , the distribution is symmetric and uniform, and for positive values the skewness is positive, meaning that the probability of drawing values closer to the minimum is larger than closer to the maximum. The value of $m$ is set to 1 for $\Delta T_{p}$ and $l$, and to 3 for the gains $P_{i}$, so that low gains are favored.

A typical evolution of the objective function with the number of iterations can be seen in Fig. 9 for the third case. At the beginning, when the temperature is still high, the probabilities of accepting bad proposals is also high, which is reflected with the high oscillations; these oscillations dampen with the number of iterations, almost disappearing at the latest stages when the objective function reaches its minimum.

Each case takes about three weeks of continuous computing, with over 7,000 iterations (each iteration implies a FE run). To reduce the waiting time, the eight cases were run in parallel in a computer cluster with 12 virtual cores. A smaller number of iterations (for instance 2,500 in Fig. 9) could have been accepted, although at the risk of not reaching the global optimum.

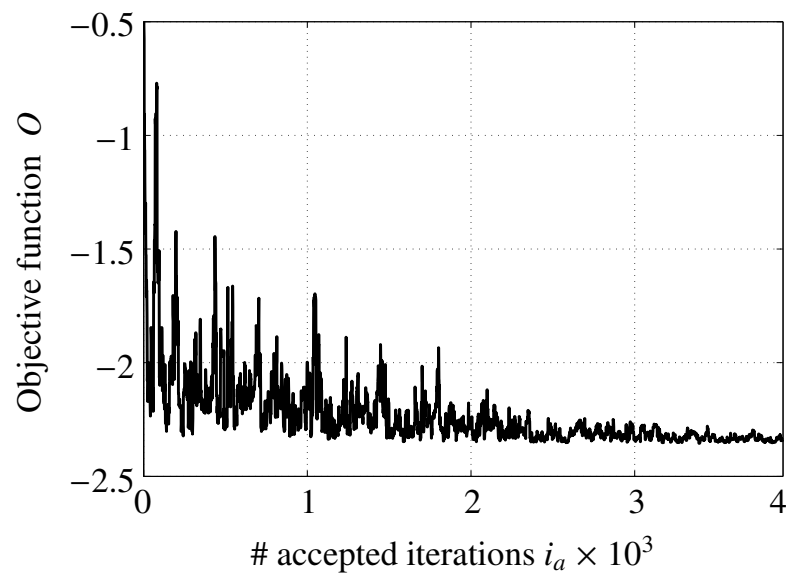

Figure 9: Evolution of the objective function $O$ vs. number of accepted iterations for the third case of Table 3.

\section{Optimized pulses}

In this section, the results of the eight cases defined in Table 3 are studied. The top plots of Figs. 10 and 11 are for the optimized pulse shape, the bottom ones for the induced $T_{c}$ evolution during the transient process. Note that the time axes span different ranges. During the optimization of each pulse, at each iteration, the prescribed $\mathrm{BC}$ are equal to those of Section 3, but the optimal intensity is analytically recalculated for the TE length at that iteration.

Any proposed parameter set that violates the criteria of maximum Tresca stress is automatically discarded. As a result, the optimal pulses not only give the best values for the selected goals, but also an admissible stress level.

\subsection{Optimized pulses for single goals}

The first case optimizes the main target of a PT: maximum overcooling $\Delta T_{p}$. The optimal pulse obtained (Fig. 10 top left) does not resemble any of the simple shapes of Fig. 5, but rather it looks like a "two-step" sequence of slightly quadratic ramps followed by a rapid descend to finish the pulse. Some resemblance with the generic pulse $\mathcal{F}$, identified as optimal in Table 1 exists, although the second flat stretch is reduced. There is an abrupt and short descend between both ramps that helps to relieve stresses.

As can be appreciated in Fig. 10 bottom left and Table 4 , the overcooling is a significant $12{ }^{\circ} \mathrm{C}$ although the Tresca stress is very close to the maximum allowable. The stress restriction is an important limiting factor in the optimization process: recall that, case $\mathcal{F}$ in the parametric analysis reaches an overcooling $16.1^{\circ} \mathrm{C}$, 

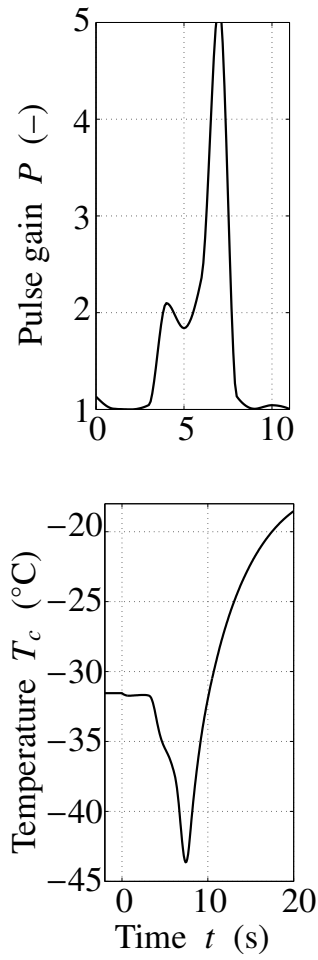
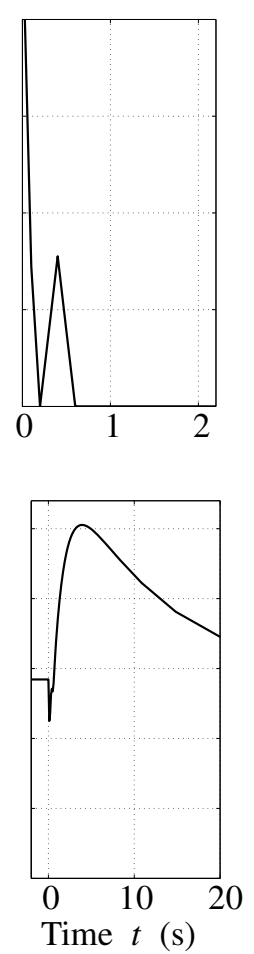
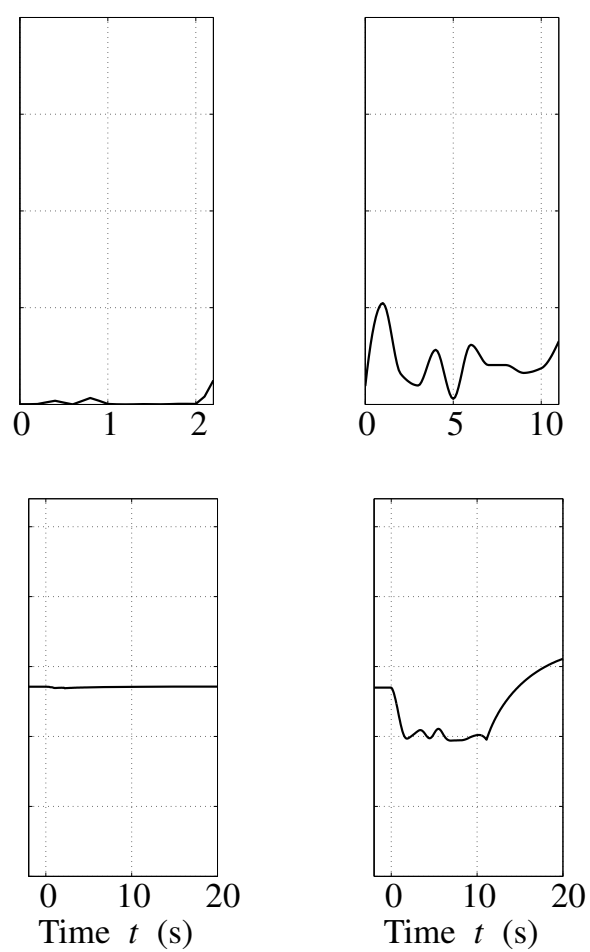

Figure 10: Optimized pulses (top) and cold face temperature distribution (bottom) with variable thermoelement length, for cases 1 to 4.

although under an inadmissible stress of $72.8 \mathrm{MPa}$. The high stress reached in the optimization is mostly due to the pulse duration, close to the highest permitted $t_{p}$, and the pulse maximum is close to the highest allowed $P$. Note that the overheating tends to be very important since this case does not take this target into account.

The minimum $T_{c}$ happens at the end of the pulse when most of the Peltier flux has accumulated and the Joule flux has not arrived yet to the cold face thanks to the smooth pulse introduction. Other reasons that limit heat flux and its arrival are: a relatively short pulse of only $4.5 \mathrm{~s}$, a maximum gain close to its possible maximum $P=6$ and a TE length close to its maximum, too.

For case 2, the SA finds the optimal pulse for the minimum time to reach maximum overcooling. It is a double impulse that, in the shortest possible time, tries to provide a very high gain of $P \approx 6$ (maximum permitted, out of the ordinate axis). The target is achieved with an extremely short $t_{m n}$ of $0.025 \mathrm{~s}$ but at the cost of a disappointing $\Delta T_{p}=3{ }^{\circ} \mathrm{C}$. Again the maximum stress is the main limiting factor, since the initial $P$ is high and the pulse is introduced abruptly; the associated optimal TE length is close to its minimum allowable value.

Such a pulse would not make much sense in real applications because it provokes the appearance of a strong and sudden Joule effect. Additionally, this shape is not good for any of the other targets as seen in Table 4.

For case 3, the SA searches for the optimal pulse with minimum overheating to find, not surprisingly, that the pulse is the absence of a pulse. The pulse is flat, and so is the temperature response. This case serves as a validation of the algorithm rather than being a practical result.

For case 4, the SA looks for the pulse that holds the overcooling as long as possible. The optimized shape is a succession of three irregular subpulses with low gain $P<2$ since a low $\Delta T_{p}=3.9^{\circ} \mathrm{C}$ makes it easier to maintain the overcooling. The first subpulse has the highest relative $P$ to quickly reach the maximum overcooling as in case 2. The other two subpulses come into action at the instants when $T_{c}$ looses the $80 \%$ of $\Delta T_{p}$. These two subpulses present a lower $P$ to achieve an approximate constant $T_{c}$ evolution: not as much energy is needed now to maintain the $\Delta T_{p}$ as in the first subpulse. The final $t_{h}$ is a compelling $9.8 \mathrm{~s}$ or $90 \%$ of the total pulse duration.

The maximum stress is low since the gain is also low and introduced smoothly, and the optimal length is close to its allowable maximum to retard the arrival of overheating; the pulse duration is the longest permitted. 

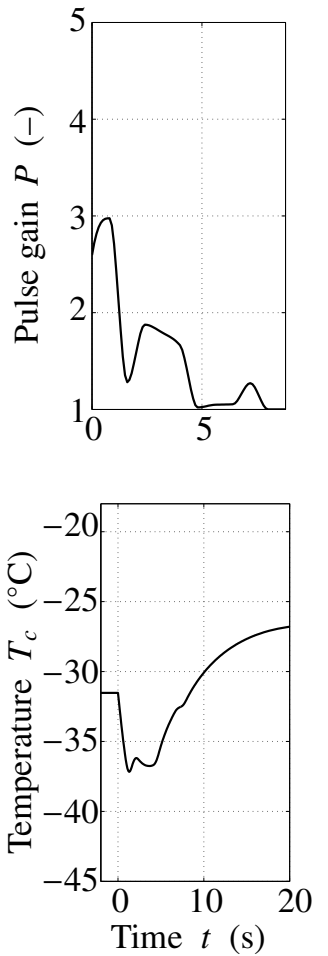
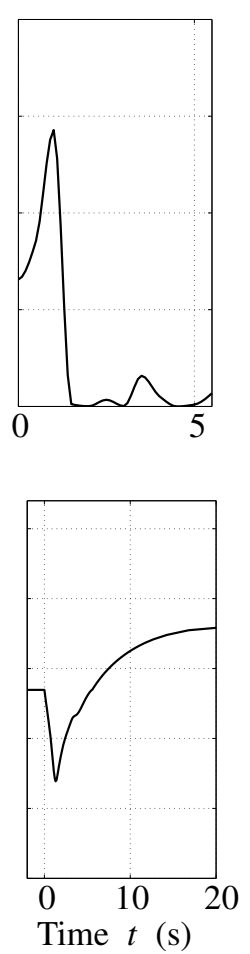
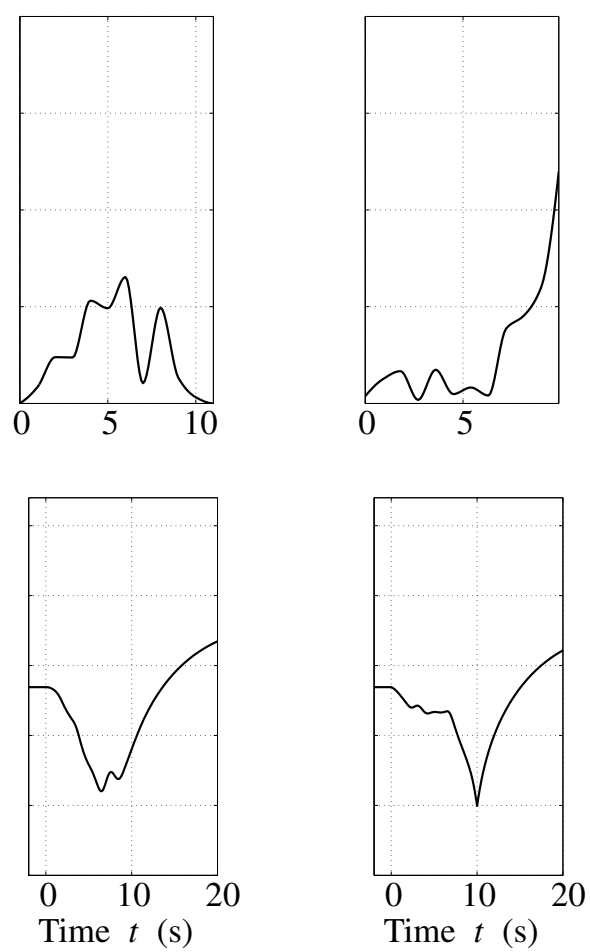

Figure 11: Optimized pulses (top) and cold face temperature evolution (bottom) with variable thermoelement length, for cases 5 to 8.

\subsection{Optimized pulses for several goals}

In this subsection, four cases combining several of the previous single goals are presented. In all of them, the optimal pulse will attempt, at least, to attain maximum overcooling $\Delta T_{p}$ and minimum overheating $\Delta T_{p p}$ and the different goals are equally weighted in their contribution to the optimization function.

For case 5, the SA finds the optimal pulse jointly accounting for all targets. The resulting pulse is rather complicated, in particular with a high gain at the pulse beginning to reduce $t_{m n}$ followed by other smaller subpulses to increase $t_{h}$. To keep $\Delta T_{p p}$ at bay, this pulse must not increase the Joule effect much above that of the steady state, something that can be achieved decreasing $P$ quickly after the first peak.

Analyzing in detail the pulse, it can be observed that the gain of the first subpulse is higher than that of the second one, inducing a sharp $T_{c}$ descend and moderating the stresses before reaching an acceptable $\Delta T_{p}$. An interesting point is that the pulse does not start with the maximum gain although climbs to it quickly; this solution is a trade-off between targets $\Delta T_{p}$ and $t_{m n}$. After the first subpulse, the targets that control the pulse shape are the minimization of $\Delta T_{p p}$ and the maximization of $t_{h}$. The optimal length of the TE is high although not at its maximum since the pulse tries to minimize $t_{m n}$. The pulse duration is long to favor $t_{h}$, since the extra energy to maintain the overcooling is low given that the gains are low.

From these results it is clear that there is an important trade-off between $t_{m n}$ and $t_{h}$, making difficult to obtain a pulse with values close to their optimal when each target is considered individually (see Table 4). It will be seen next that removing the minimization of $t_{m n}$ from the optimization function, improves significantly the optimal values of the rest of the goals.

For case 6, the SA looks for the optimal pulse accounting for all the targets except the maximization of the holding time. The resulting pulse has a first subpulse like in the previous case, but since $t_{h}$ is not a target the maximum $P$ is higher, which improves $\Delta T_{p}$. For the same reason, the second subpulse observed in the previous case is missing and, consequently, the total pulse is shorter and $\Delta T_{p p}$ smaller than in case 5 .

For case 7 , the SA analyzes a more practical case in which all targets are optimized jointly except the minimization of $t_{m n}$. The resulting pulse starts at $P=1$, progresses smoothly as in case 1 but with a limit since minimizing $\Delta T_{p p}$ and maximizing $t_{h}$ are also sought. After three discontinuous ramps, a shorter subpulse follows, 


\begin{tabular}{|c|c|c|c|c|c|c|c|c|c|c|}
\hline Case & $\Delta T_{p}$ & $t_{m n}$ & $\Delta T_{p p}$ & $t_{h}$ & $P_{m x}$ & $t_{p}$ & $l$ & $\mathcal{T}_{t r}$ & $O$ & Ratio \\
\hline & ${ }^{\circ} \mathrm{C}$ & $\mathrm{s}$ & ${ }^{\circ} \mathrm{C}$ & $\mathrm{s}$ & - & $\mathrm{s}$ & $\mathrm{mm}$ & $\mathrm{MPa}$ & - & - \\
\hline \hline 1 & $\mathbf{1 2 . 1}$ & 7.3 & 14.4 & 1.1 & 5.2 & 11 & 6.9 & 59.4 & -1.2 & 1.9 \\
\hline 2 & 3.1 & $\mathbf{0 . 0 3}$ & 11.1 & 0.001 & 5.9 & 2.2 & 2.5 & 59.5 & -1.6 & 121 \\
\hline 3 & 0.1 & 2.1 & $\mathbf{0 . 0 1}$ & 0.46 & 1.25 & 2.2 & 5.6 & 23.0 & -2.4 & 985 \\
\hline 4 & 3.8 & 6.8 & 2.9 & $\mathbf{9 . 8}$ & 2 & 11 & 6.4 & 28.9 & -1.8 & 12 \\
\hline 5 & $\mathbf{5 . 6}$ & $\mathbf{1 . 2}$ & $\mathbf{4 . 9}$ & $\mathbf{3 . 8}$ & 3 & 8.8 & 6.6 & 33.6 & -0.9 & $0.9 / 2.5 / 2.4 / 4.7$ \\
\hline 6 & $\mathbf{6 . 5}$ & $\mathbf{1 . 2}$ & $\mathbf{4 . 5}$ & 0.75 & 3.9 & 5.5 & 6.7 & 35.2 & -0.8 & $1.0 / 2.5 / 2.6 /-$ \\
\hline 7 & $\mathbf{7 . 4}$ & 6.4 & $\mathbf{4 . 5}$ & $\mathbf{3 . 8}$ & 2.3 & 11 & 6.9 & 33.8 & -1.1 & $1.2 /-/ 2.6 / 4.6$ \\
\hline 8 & $\mathbf{8 . 5}$ & 9.9 & $\mathbf{4 . 3}$ & 0.96 & 3.4 & 9.9 & 7.0 & 35.4 & -1.1 & $1.3 /-/ 5.3 /-$ \\
\hline
\end{tabular}

Table 4: Optimization of pulses for the eight cases, see Table 3 for corresponding weights. Definitions in Figs. 1 and 2 . Last column shows up to four values with the ratio between the target(s) value(s) obtained for the optimal pulse and the corresponding target(s) value(s) computed with the average parameters in Table 2 . The target values that are subject to optimization for each case are shown in bold.

which maintains $t_{h}$. The long duration of the pulse is compensated by low values of $P$ to limit the pulse total energy. The maximum overcooling $\Delta T_{p}$ reaches an interesting $7.5^{\circ} \mathrm{C}$ with an acceptable overheating $\Delta T_{p p}$ of $4.5^{\circ} \mathrm{C}$. This solution is comparable to pulse $\mathcal{D}$ from Fig. 5 that gave good results for the three targets, see Table 1.

Finally, for case 8 , the SA looks for a pulse producing maximum overcooling and minimum overheating. The resulting pulse has two initial peaks as in case 1 , but with smaller $P$ and slightly lower duration. It ends with a high peak, resulting in the maximum overcooling and minimum overheating of all of the multi-goal cases analyzed. Not taking into account the low-gain train at the beginning, this shape is similar to $\mathcal{B}$ from Fig. 5. In the last two cases the length of the TE is almost maximum since $t_{m n}$ is not considered in the optimization.

\subsection{Discussion}

From a practical point of view, the optimal pulses obtained by SA could be post-processed with "engineering" sense. For case 1, the optimal pulse show two small subpulses before and after the main double pulse that could be removed. Also in this case, a flat transition from the first subpulse to the second would give basically the same results. For case 2, the second "spike" could be removed not affecting much the final results. For cases 5 and 6 the small subpulses after $5 \mathrm{~s}$ are almost irrelevant due to the small energy that they introduce (they only have some influence in the value of $\left.\Delta T_{p p}\right)$. Note also that the probability distribution of $P_{i}$ has higher densities for (and therefore higher chances to draw) low gain values.

Table 4 shows the values of the different targets, the maximum pulse gain, the total pulse duration, the TE length, the maximum Tresca stress and the final $O$, for the eight cases. A bold font has been used to highlight the target that is subject to optimization for each case. The last column shows up to four values associated to the targets being analyzed for each case. These values are the ratio of the optimal result obtained with respect to the result obtained when the mean values of the parameters in Table 2 are used. Besides the trivial case 3 and the not very practical case 2 , these ratios show important improvements.

The TE internal distribution of stresses was studied in detail in [14] for constant pulses and it is important for the understanding of general pulses. Recall that the parameters that are specially important for the generation of stresses inside the TE are:

- The TE length $l$.

- The maximum $P$, specially at the pulse beginning.

- The pulse duration $t_{p}$.

Indeed, if $l$ is short, most of the TE cannot expand freely in the plane $x_{1}-x_{3}$ and the ends are highly loaded due to the restrictions with other materials and with the mechanical BC. Tresca stress increases linearly, proportionally to the initial $P$, at the beginning and then tends towards an asymptote; however, after $t_{p}$, the stress growth stops slowly returning to that of steady state.

Contour maps inside the TE for cases 1, 2, 5, 7 and 8 are shown in Fig. 12. The Tresca stresses are plotted, for each case, at the instant when they are maximum. The high stress appears at the faces in contact with other materials, (thin solder, conducting $\mathrm{Cu}$ ) and concentrates at the lower left corner. For all cases except 2, the rest of the TE is approximately under free expansion and develops strains instead of stresses. The stress distributions 
and their maximums are similar for cases 1,2 on one side and 5, 7, 8 on the other, with maximums higher in the first two cases. These similarities are due to equal $\mathrm{BC}$ and analogous energies; for cases 1 and 2 they are also due to a comparable combination between energy and pulse slope at the beginning of the pulse.

\section{Conclusions}

Optimal pulse shapes and durations, together with optimal thermoelement lengths have been obtained for a variety of single and combined goals, which include maximum overcooling, minimum overheating, minimum time to maximum overcooling and maximum holding time. After a parametric study to understand how different simple pulse shapes influence the values of the different goals, simulated annealing optimization allows to determine the optimal pulses of irregular shapes.

Several main conclusions can be established:

- Multiobjective optimizations with relatively few parameters are computationally costly since nonlinear, dynamic, coupled numerical methods and heuristic optimization algorithms must be used.

- Non-regular pulse shapes can substantially improve the performance of pulsed thermoelectrics, but the stresses can be so high as to cause failure of the device.

- There is an important trade-off between the different goals. No pulse can attain very good values for all goals when compared with the values obtained for pulses optimal for a single goal.

- Optimizations with combined targets are necessary for practical applications, the choice of the targets will influence substantially the final shape of the optimal pulse.

\section{Acknowledgments}

This research was partially supported by the grants, Haut-de-France Region (CR Picardie, 1202015-RDISTRUCT-000010), EU funding (FEDER, RDISTRUCT-000010) for Chaire-de-Mécanique, and Spanish Ministry of Economy and Competitiveness grant CGL2014-59841-P. These supports are gratefully acknowledged.

\section{References}

[1] G.J. Snyder, J.P. Fleurial, and T. Caillat. Supercooling of Peltier cooler using a current pulse. Journal of Applied Physics, 92(3):1564-1569, 2002.

[2] Iván Rivera, Aldo Figueroa, and Federico Vázquez. Optimization of supercooling effect in nanoscaled thermoelectric layers. Communications in Applied and Industrial Mathematics, 7(2):98-110, 2016.

[3] Hao Lv, Xiao-Dong Wang, Tian-Hu Wang, and Jing-Hui Meng. Optimal pulse current shape for transient supercooling of thermoelectric cooler. Energy, 83:788-796, 2015.

[4] Ming Ma and Jianlin Yu. A numerical study on the temperature overshoot characteristic of a realistic thermoelectric module under a current pulse operation. International Journal of Heat and Mass Transfer, 72:234-241, 2014.

[5] M. Ma and J. Yu. Experimental study on transient cooling characteristics of a realistic thermoelectric module under a current pulse operation. Energy Conversion and Management, 126:210 $-216,2016$.

[6] H.Y. Zhang, Y.C. Mui, and M. Tarin. Analysis of thermoelectric cooler performance for high power electronic packages. Applied Thermal Engineering, 30:561 - 568, 2010.

[7] D. Astrain, J.G. Vián, and M. Domínguez. Increase of cop in the thermoelectric refrigeration by the optimization of heat dissipation. Applied Thermal Engineering, 23:2183-2200, 2003.

[8] Limei Shen, Zhilong Tu, Qiang Hu, Cheng Tao, and Huanxin Chen. The optimization design and parametric study of thermoelectric radiant cooling and heating panel. Applied Thermal Engineering, 112:688 - 697, 2017.

[9] M. Manno, Peng Wang, and A. Bar-Cohen. Pulsed thermoelectric cooling for improved suppression of a germanium hotspot. Components, Packaging and Manufacturing Technology, IEEE Transactions on, 4(4):602-611, 2014.

[10] Fankai Meng, Lingen Chen, and Fengrui Sun. Performance optimization for two-stage thermoelectric refrigerator system driven by two-stage thermoelectric generator. Cryogenics, 49(2):57$65,2009$.

[11] H. Lv, X.-D. Wang, J.-H. Meng, T.-H. Wang, and W.-M. Yan. Enhancement of maximum temperature drop across thermoelectric cooler through two-stage design and transient supercooling effect. Applied Energy, 175:285 - 292, 2016.

[12] S. Lin, M. Ma, J. Wang, and J. Yu. Experiment investigation of a two-stage thermoelectric cooler under current pulse operation. Applied Energy, 180:628 - 636, 2016.

[13] Q Zhou, Z Bian, and A Shakouri. Pulsed cooling of inhomogeneous thermoelectric materials. Journal of Physics D: Applied Physics, 40(14):4376, 2007.

[14] J.L. Pérez-Aparicio, R. Palma, and P. Moreno-Navarro. Elastothermoelectric non-linear, fully coupled, and dynamic finite element analysis of pulsed thermoelectrics. Applied Thermal Engineering, 107:398-409, 2016.

[15] J.L. Pérez-Aparicio, R. Palma, and R.L. Taylor. Multiphysics and thermodynamic formulations for equilibrium and nonequilibrium interactions: non-linear finite elements applied to multi-coupled active materials. Archives of Computational Methods in Engineering, 23(3):535-583, 2016.

[16] R. Palma, J.L. Pérez-Aparicio, and R.L. Taylor. Non-linear finite element formulation applied to thermoelectric materials under hyperbolic heat conduction model. Computer Method in Applied Mechanics and Engineering, 213:93-103, 2012.

[17] R. Palma, J.L. Pérez-Aparicio, and R. Bravo. Study of hysteretic photovoltaic behavior using the finite element method, extended thermodynamic and inverse models. Energy Conversion and Management, 65:557-563, 2013. 

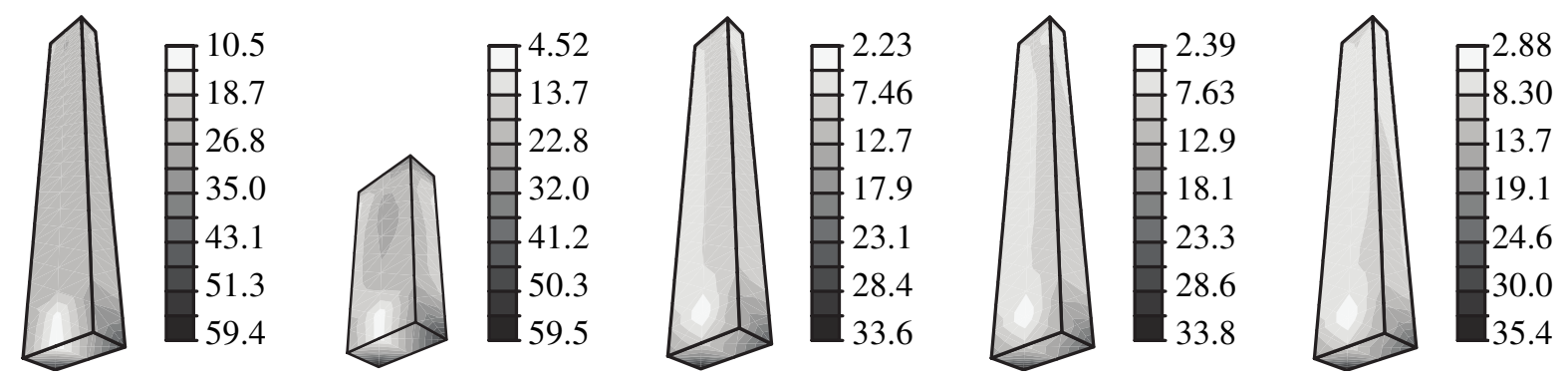

Figure 12: Contour plots of Tresca equivalent stresses in MPa for thermoelements of cases 1, 2, 5, 7 and 8, see Table 2. Three dimensional finite elements with meshes similar to that of Fig. 2.

[18] R.L. Taylor. FEAP A Finite Element Analysis Program: User Manual. University of California, Berkeley, 2010. http://www.ce.berkeley.edu/feap.

[19] J.L. Pérez-Aparicio, R. Palma, and R.L. Taylor. Finite element analysis and material sensitivity of Peltier thermoelectric cells coolers. International Journal of Heat and Mass Transfer, 55:1363-1374, 2012.

[20] P. Moreno-Navarro, A. Ibrahimbegovic, and J.L. PérezAparicio. Plasticity coupled with thermo-electric fields: Thermodynamics framework and finite element method computations. Computer Methods in Applied Mechanics and Engineering, 315:50 - 72, 2017

[21] O.C. Zienkiewicz, R.L. Taylor, and J.Z. Zhu. The Finite Element Method: The Basis, 7th ed. Elsevier Butterworth-Heinemann, 2013.

[22] Dongliang Zhao and Gang Tan. A review of thermoelectric cooling: Materials, modeling and applications. Applied Thermal Engineering, 66(1-2):15-24, 2014.

[23] J.N. Mao, X. Chen H, H. Jia, and X.L. Qian. The transient behavior of peltier junctions pulsed with supercooling. Journal of Applied Physics, 112:014514-9, 2012.

[24] D. Mitrani et. al. Transient distributed parameter electrical analogous model of te devices. Microelectronics Journal, 40(9): 1406-1410, 2009.

[25] Th. Clin, S. Turenne, D. Vasilevskiy, and R.A. Masut. Numerical simulation of the thermomechanical behavior of extruded bismuth telluride alloy module. Journal of Electronic Materials, 38(7):994-1001, 2009.

[26] C.L. Deutsch and A.G. Journel. Geostatistical Software Library and User's Guide. Oxford University Press, 1998.

[27] E. Aarts and J. Korst. Simulated Annealing and Boltzmann Machines: A Stochastic Approach to Combinatorial Optimization and Neural Computing. Wiley Interscience Series in Discrete Mathematics and Optimization. John Wiley \& Sons, Chichester, 1989.

[28] Mathworks. Documentation center. http://es.mathworks.com/help/matlab/. 